\title{
Review
}

\section{Overview of intra-uterine insemination in a Sri Lankan scenario}

\author{
Deepal S Weerasekera ${ }^{1}$ \\ Sri Lanka Journal of Obstetrics and Gynaecology 2010; 32: 17-18
}

In-vitro fertilization services are still not available in the government sector in Sri Lanka and there are no indicators of these services being available in the government sector in the near future. Even in the private sector these services are available only in a few centres at a considerable cost. Like in many developing countries in Sri Lanka also the cost of IVF treatment is a major issue. Most of the health insurance policies do not cover expenses involved in subfertility treatment. Proper evaluation of the couple is extremely important before planning treatment.

Compared to IVF treatment intra-uterine insemination (IUI) is a much simpler method which is highly cost effective and can be easily popularize in the government sector health institutions. But certainly this is not the first line of treatment as ovulation monitoring and timed intercourse should be tried before initiating IUI therapy in suitable couples. IUI should always be done with washed prepared sperms. Use of neat sperms for IUI can result in painful uterine cramps, collapse, infections and therefore should never be performed. Artificial insemination can be done in several ways i.e. intra-vaginal, intracervical, intrafallopian, intraperitoneal or intrauterine. The rationale for the use of IUI instead, intra-vaginal or intra-cervical insemination is to reduce the effect of factors such as vaginal acidity and cervical mucus hostility. Also there are benefits from deposition of prepared motile morphologically normal sperms as close as possible to the oocytes at the time of ovulation. Ejaculatory failure is a definitive indication for IUI. In cervical mucus hostility IUI bypasses the mucus in the cervical canal. Other indications for IUI are oligospermia, and unexplained infertility.

\section{IUI in natural and stimulated cycles}

IUI can be performed either in a natural or a stimulated cycle. Clomiphene citrate is the most commonly used agent for ovarian stimulation. Tamoxifen and aromatase inhibitors (letrazole) are used in women who do not respond to clomiphene citrate.

\footnotetext{
${ }^{1}$ Clinical Director, Prarthana Center for ART, Rajagiriya Colombo.
}

If there is no success with oral therapy gonadotrophins are used. The rationale for the use of ovarian stimulation is to increase the number of oocytes available for fertilization and thus to improve the chance of pregnancy. Human chorionic gonadotrophin (hCG) is usually used at the end of the stimulation phase to achieve final maturation of the oocyte. Stimulation enhances steroid production which may improve the chance of fertilization and embryo implantation ${ }^{1}$. This potential increase in success rates must be balanced against the increased cost of the medication, necessity for monitoring, potential complications of these medications including ovarian hyperstimulation syndrome and multiple pregnancy ${ }^{2}$. Timing of ovulation is important to perform the IUI at the correct time. Early methods used for this purpose such as the measurement of basal body temperature chart and assessment of cyclical changes in cervical mucus are no longer practiced due to their inaccuracy and practical difficulties. Detection of urinary LH surge and ultrasound assessment of follicular growth and rupture are the most accurate methods of monitoring ovulation. The use of a "dip stick" LH test kit can predict ovulation in over $80 \%$ of cycles $^{3}$. If hCG is administered when the diameter of the leading follicle is $18-20 \mathrm{~mm}$ ovulation may be expected to occur 34-40 hours later ${ }^{4}$. The maximum number of times IUI can be performed is still debatable. The common sense of opinion at present is to resort to IVF if there is no success after six cycles of IUIs

\section{Sperm preparation}

There are several different sperm preparation techniques for IUI.

- Standard sperm wash (SSW) - removes seminal plasma from the semen specimen by centrifugation.

- Swim-up (SWU) - uses self migration of motile sperms from the bottom fraction to the top fraction followed by centrifugation to remove dead and immotile sperms and debris.

- Swim-down (SWD) - self migration of motile sperms from the top to the bottom fraction of media which contains increasing concentrations of human serum albumin. 
- Density gradient method (DGC) - separates motile sperms by density, using repeated centrifugation of semen mixed with a media containing a colloidal suspension of silica products. Motile sperms have higher density than non-motile and dead sperms.

Sperm preparation using the density gradient technique yields the highest number of motile sperms when compared with simple washing or swim-up or swim-down methods. This method also significantly reduces bacterial contamination ${ }^{5}$. In the treatment of unexplained subfertility pregnancy rate achieved from super-ovulation alone was less than when combined with IUI ${ }^{6}$. In the treatment of male subfertility, ovulation induction with IUI gave better results than ovulation induction alone ${ }^{7}$.

\section{Complications of treatment}

Painful uterine contractions which may occur during insemination can usually be minimized by inseminating slowly. Pelvic sepsis is a serious complication which occurs rarely as you need to send the catheter through the natural cervical barrier. Unwashed semen should never be used for insemination as this can cause serious anaphylaxis. Multiple pregnancy and ovarian hyperstimulation are two complications which the patients need to be counselled before the procedure. These complications can be minimized by monitoring of treatment cycles and if necessary abandoning the cycle or converting it to an IVF cycle.

\section{Conclusions}

IUI is an effective, non-invasive relatively simple and cost effective method of treatment for certain diagnostic groups of infertile couples. Unfortunately in our hospitals this procedure has not been given its due place yet. To provide a good quality IUI service there should be adequate facilities for semen preparation, cycle monitoring and well trained staff for sperm preparation. The main advantages of IUI over IVF are its simplicity and relative inexpensiveness. This needs to be considered seriously in prioritizing the health-care needs in a cost-effective basis in a country such as Sri Lanka where resources for health care are limited.

\section{References}

1. Wallach EE. Gonadotropin treatment of the ovulatory patient; the pros and cons of empiric therapy for infertility. Fertil Steril 1991; 55: 478-80.

2. Levene MI, Wild J, Steer P. Higher multiple births and the modern management of infertility in Britain. The British Association of Perinatal Medicine. Br. J. Obstet. Gynaecol. 1992; 99: 607-13.

3. Vermesh M, Kletzky OA, Davajan V, Israel R. Monitoring techniques to predict and detect ovulation. Fertil Steril 1987; 47: 259-64.

4. Brinsden PR, Dickay RP. An overview of intrauterine insemination and ovulation induction. In: Dickey RP, Brinsden PR, Pyrzak R. eds. Manual of intrauterine insemination and ovulation induction. Cambridge University Press 2010: 3.

5. Punjabi U, Gerris J, Van Bijlen J, et al. Comparison between different pre-treatment techniques for sperm recovery prior to intra-uterine insemination, GIFT or IVF. Hum. Reprod 1990; 5; 75-83.

6. Crosignani PG, Walters DE. Clinical pregnancy and male subfertility: the ESHRE multicentre trial on the treatment of male subfertility. Hum. Reprod 1994; 9: 1112-18.

7. Crosignani PG, Walters DE, Soliani A. The ESHRE multicentre trial on the treatment of unexplained infertility: a preliminary report. Hum Reprod 1991; 6: 953-8. 